\title{
Colorectal cancer and self-reported tooth agenesis
}

\author{
Noralane M Lindor ${ }^{1 *}$, Aung Ko Win², Steven Gallinger ${ }^{3}$, Darshana Daftary ${ }^{3}$, Stephen N Thibodeau ${ }^{4}$, \\ Renato Silva ${ }^{5,6}$ and Ariadne Letra ${ }^{5,6}$
}

\begin{abstract}
Background: Germline mutations in APC and AXIN2 are both associated with colon neoplasia as well as anomalous dental development. We tested the hypothesis that congenitally missing teeth may occur more commonly in individuals diagnosed with colorectal cancer than in individuals without this diagnosis.

Methods: Via a survey conducted on 1636 individuals with colorectal cancer (CRC) and 2788 individuals with no colorectal cancer from the Colon Cancer Family Registry, self-reported information on congenitally missing teeth was collected. The frequency of missing teeth between cases and controls was compared using Pearson's chi-squared test or Fisher's exact test.

Results: $4.8 \%$ of cases and $5.7 \%$ of controls reported having at least one missing tooth $(p=0.20)$. When we stratified by recruitment site, gender, and mutation status where available, frequency of missing teeth was not statistically significantly different between cases and controls.

Conclusions: This study did not provide support for there being a general predisposition to missing teeth among a large cohort of CRC patients. The study neither addresses nor excludes the possibility, however, that individuals presenting with notable hypodontia/oligodontia might still have an increased risk for colorectal neoplasia.
\end{abstract}

Keywords: AXIN2, Colorectal neoplasia, Hypodontia, Oligodontia

\section{Background}

Teeth are formed from neural crest-derived mesenchyme and epithelium of the first branchial arch and part of the frontonasal process [1,2]. The main proteins involved in development of dentition belong to one of five signaling pathways including Notch, bone morphogenetic protein, fibroblast growth factor, sonic hedgehog, and Wingless/ integration (WNT).

A Finnish family was described in 2004 [3] in which a nonsense mutation in axis inhibition protein 2 (AXIN2) was found to co-segregate with an oligodontia (severe tooth agenesis) phenotype. Further characterization of the family identified adenomatous colorectal polyps and cancer as part of the phenotype. A second family, also in Finland, with the same AXIN2 mutation was reported by Renkonen et al. [4] as part of their study of 29 polyposis families in which no adenomatous polyposis coli $(A P C)$ mutation was found. The $A P C$ gene, mutations of which cause Familial Adenomatous Polyposis, is a key component

\footnotetext{
* Correspondence: nlindor@mayo.edu

'Department of Health Sciences Research, Mayo Clinic, 13400 East Shea Blvd., Scottsdale, AZ 85259, USA

Full list of author information is available at the end of the article
}

of the WNT-signaling pathway. Like AXIN2, APC mutations can be associated with dental anomalies including missing or supernumerary teeth [5].

The reported association between AXIN2 variants, colorectal cancer, and tooth agenesis suggests that these conditions may be associated. However, no large-scale studies of colorectal cancer (CRC) patients have been conducted to assess a general association between colorectal neoplasia and missing teeth. The aim of this study was to determine if congenitally missing teeth may occur more commonly in individuals diagnosed with colorectal cancer than in individuals without this diagnosis. If so, then questioning people about missing teeth might help identify those at increased risk for colorectal cancer. In this study, we surveyed participants of the Colon Cancer Family Registry (C-CFR) for self-reported missing permanent teeth and compared findings with unaffected relatives and controls. We also assayed for the presence of AXIN2 mutations in the only two probands presenting with colon cancer and tooth agenesis, and in family members with tooth agenesis but not cancer. 


\section{Methods}

Participants in this study were recruited through the C-CFR, a National Institutes of Health-supported consortium established to provide resources for the interdisciplinary study of the etiology of colon cancer and identification of at-risk populations who may benefit from translational research and current therapeutic strategies. The consortium consists of six research centers: Fred Hutchinson Cancer Research Center, Seattle, Washington; University of Hawaii Cancer Research Center, Honolulu, Hawaii; Mayo Clinic, Rochester, Minnesota; The University of Southern California Consortium, Los Angeles, California; Cancer Care Ontario, Ontario, Canada; and the University of Melbourne, Victoria, Australia. Detailed information about the C-CFR is described by Newcomb et al. [6]. This study included only CRC families from the Mayo Clinic and Toronto C-CFR sites, recruited both through the clinics and from population-based sources. After enrollment of the proband, recruitment invitation was extended to adult first- and second-degree relatives and in some families beyond that. Both Mayo Clinic and Cancer Care Ontario systematically oversampled for multiplex families and young-onset probands, but no information about dentition was known prior to enrollment. For C-CFR study participants with CRC, tumor characterization is routinely conducted to determine DNA mismatch repair status, and if abnormal, further testing is conducted for germline mutations in DNA mismatch repair genes [7]. Genetic testing for mutations in MUTYH is also standard for C-CFR probands [8]. Results of this molecular characterization were not used as inclusion or exclusion criteria for this study.

Standardized protocols were used to collect family history, risk factor information, and biospecimens. For this study, two questions were added to the 5- or 10year follow-up survey. The first asked if the participant had any permanent teeth that never formed, not counting wisdom teeth. If they answered yes, the second question asked how many teeth were missing. Cases were defined as probands and relatives who had been diagnosed with colorectal cancer. Controls were defined as relatives and spouses who had never been diagnosed with any cancer. All study participants provided written informed consent and institutional review board approval was obtained at each center.

Five of the CRC cases with a missing tooth reported one or more relatives with missing teeth (none of those relatives had CRC). On the two Mayo probands and their five relatives, we performed direct sequencing of all AXIN2 exons and exon-intron boundaries to look for mutations (DNA not available on the three multiplex Toronto families). Sequencing reactions were performed according to established protocols. Primer sequences and reaction conditions are available upon request.
The frequency of missing teeth between cases and controls were compared using Pearson's chi-squared test or Fisher's exact test. The comparisons were also made stratified by the recruitment site (Cancer Care Ontario, Mayo Clinic), gender (male, female), and germline mutation status (MLH1, MSH2, MSH6, PMS2n MUTYH). Note: the germline status of these genes was available in the C-CFR. These genes are not suspected of being involved with dental formation but were included in a stratified analysis just to be certain they were not contributing anything to the phenotype. Genetic testing for $A P C$ and AXIN2 were not conducted systematically in the C-CFR so were not part of this study design nor stratified analyses. However, sequencing of AXIN2 was conducted on two individuals who did have a colon cancer/missing teeth phenotype, and so this was reported, though it was not a primary aim of the study. All statistical analyses were conducted using STATA 12.1 [StataCorp. Stata Statistical Software: Release 12. College Station, TX: StataCorp LP; 2011].

\section{Results}

Of a total of 1809 cases (1596 probands and 213 relatives), we excluded 173 (148 probands and 25 relatives) who did not provide missing teeth information. Of a total of 3005 controls (2737 relatives and 268 spouses), we excluded 217 (207 relatives and 10 spouses) who did not provide missing teeth information. The remaining 1636 cases (1448 probands and 188 relatives) and 2788 controls (2530 relatives and 258 spouses) entered into the analysis. Table 1 shows the baseline characteristics of the cases versus the controls.

We observed that $4.8 \%$ of cases and $5.7 \%$ of controls reported having at least one missing tooth (Table 1), and there was no statistical evidence of difference between them $(p=0.20)$. When we stratified by recruitment site, gender, and mutation status where available, frequency of missing teeth was not statistically significantly different between cases and controls. Women were reported to have more missing teeth than men in both cases (odds ratio $[\mathrm{OR}]=2.21$; $95 \%$ confidence interval $[\mathrm{CI}]=1.30-3.93$; $\mathrm{p}=0.002)$ and controls $(\mathrm{OR}=1.56 ; 95 \% \mathrm{CI}=1.09-2.27$; $\mathrm{p}=0.01$; Table 2).

We looked to see if there were families in which multiple individuals reported missing teeth, and there were only a few. There were five CRC cases with a missing tooth reported in which one or more relatives had missing teeth (none of those relatives had CRC); eight of the CRC cases who themselves did not have missing teeth had two or more relatives with missing teeth; and of the CRC cases who did report one or more missing teeth, 24 had no other relatives surveyed in this study. All other probands had no reported missing teeth and 0-1 relatives with missing teeth. 
Table 1 Self-reported missing teeth in study participants

\begin{tabular}{llll}
\hline & & $\begin{array}{l}\text { Cases* }^{*} \\
(\mathbf{n}=\mathbf{1 6 3 6})\end{array}$ & $\begin{array}{l}\text { Controls }^{\dagger} \\
(\mathbf{n}=\mathbf{2 7 8 8})\end{array}$ \\
\hline Missing teeth & No & 1558 & 2630 \\
& Yes & $78(4.8 \%)$ & $158(5.7 \%)$ \\
Number of missing teeth & & & \\
& 1 & 24 & 57 \\
& 2 & 22 & 54 \\
& 3 & 2 & 6 \\
& 4 & 7 & 9 \\
& 5 & 0 & 2 \\
$\geq 6$ & 1 & 6 \\
& unknown & 22 & 24 \\
\hline
\end{tabular}

${ }^{*}$ Cases had diagnosis of colorectal cancer.

${ }^{+}$Controls had no colorectal cancers.

Direct sequencing of the two Mayo probands and their five relatives with hypodontia revealed the presence of a common P50S missense variant in exon 10 of the AXIN2 gene in one of the two probands with colon cancer and tooth agenesis, in four siblings with tooth agenesis but not colon cancer, and in one unaffected sibling (no colon cancer and no tooth agenesis). No AXIN2 mutations were found in the second proband.

Table 2 Frequency of missing teeth between cases and controls

\begin{tabular}{llll}
\hline & $\begin{array}{l}\text { Cases } \\
\text { n/N (\%) }\end{array}$ & $\begin{array}{l}\text { Controls } \\
\mathbf{n} / \mathbf{N}(\%)\end{array}$ & p value \\
\hline Total & $78 / 1636(4.8 \%)$ & $158 / 2788(5.7 \%)$ & 0.20 \\
Recruitment site & & & \\
Ontario & $51 / 928(5.5 \%)$ & $88 / 1484(5.9 \%)$ & 0.66 \\
Mayo Clinic & $27 / 708(3.8 \%)$ & $70 / 1304(5.4 \%)$ & 0.12 \\
Gender & & & \\
Male & $20 / 695(2.9 \%)$ & $47 / 1094(4.3 \%)$ & 0.12 \\
Female & $58 / 941(6.2 \%)$ & $111 / 1694(6.6 \%)$ & 0.70 \\
Mutation status* & & & \\
MLH1 & $1 / 63(1.6 \%)$ & $2 / 19(10.5 \%)$ & 0.13 \\
MSH2 & $6 / 72(8.3 \%)$ & $3 / 35(8.6 \%)$ & 0.99 \\
MSH6 & $0 / 8(0 \%)$ & $2 / 5(40 \%)$ & 0.13 \\
PMS2 & $0 / 10(0 \%)$ & $0 / 6(0 \%)$ & $N A$ \\
All MMR genes & $7 / 153(4.6 \%)$ & $7 / 65(10.8 \%)$ & 0.13 \\
MUTYH & $0 / 23(0 \%)$ & $1 / 24(4.2 \%)$ & 0.99
\end{tabular}

$\mathrm{n}=$ number of study participants with missing teeth.

$\mathrm{N}=$ total number of study participants.

*The germline status of these genes was available in the Colon Cancer Family

Registry. These genes are not suspected of being involved with dental formation but were included in a stratified analysis just to be certain they were not contributing to the phenotype.

\section{Discussion}

Prompted by the discovery of an association between colon polyps and cancer with tooth agenesis, several investigators have examined the potential for a colon neoplasia predisposition and WNT-signaling pathways genes from different perspectives. Peterlongo et al. [9] searched for AXIN2 mutations in 82 familial (CRC families in which other syndromes had been ruled out. They found 29 DNA variants, none of which appeared likely to be pathogenic. Mostowska et al. [10] studied 55 Caucasians from Poland with missing teeth (nearly half had true oligodontia) and observed an increased risk of tooth agenesis in individuals carrying the AXIN2 c.956 + 16G allele $(\mathrm{OR}=2.94 ; 95 \% \mathrm{CI}=1.10-7.82 ; \mathrm{p}=0.03)$ and the c.2062 $\mathrm{T}$ allele $(\mathrm{OR}=4.01 ; 95 \% \mathrm{CI}=1.56-10.30 ; \mathrm{p}=$ 0.02). Lejeune et al. [11] studied 39 patients with multiple adenomas and no APC mutation nor DNA mismatch repair defect and found one person with two variants of uncertain significance in AXIN2. Callahan et al. [12] studied 167 individuals with tooth agenesis (majority with only 1-2 teeth missing) and found an association between missing incisors and one of three intragenic polymorphisms in the AXIN2 gene. The most significant association was for a missense mutation in exon 10 (P50S, rs2240308) with a $\mathrm{p}$-value of 0.037 , and they concluded that their work provided further evidence that AXIN2 contributes to tooth agenesis. This suggested relationship between AXIN2 and tooth agenesis also led to findings that $A X I N 2$ gene variants may have a role in cleft lip/palate in multiple populations [13], with and without the presence of tooth agenesis as part of the clinical phenotype [14]. In a study of families with cleft lip and/or palate, a family history of cancer was reported more often than in control families with higher rates of specific cancer types including colon $(\mathrm{p}<0.001)$ [15]. Most recently, Marvin et al. [16] reported a novel protein truncating AXIN2 mutation (c.1989G > A; p.Try663X) that segregated in an autosomal-dominant pattern with colonic polyposis, gastric polyps, and a mild ectodermal dysplasia phenotype.

This current study was conducted to evaluate selfreported missing teeth as a potential marker for predisposition to CRC. We did not find that participants with CRC were any more likely to report missing teeth than their unaffected relatives or unrelated controls. To not form one or more of the normal 20 deciduous teeth and 32 permanent teeth is said to be one of the most common developmental anomalies in humans.

Approximately $20 \%$ of humans will have at least one missing third molar ("wisdom teeth") and 3-10\% have failure of formation for one or more of the other permanent teeth (reviewed in Nieminen 2009 reference). Both our CRC cases and the unaffected control group reported frequencies of missing teeth consistent with this range. The term hypodontia is a term used to describe only a few 
missing teeth, while oligodontia refers to a more severe anomaly with six or more missing teeth. True oligodontia is quite rare, affecting $0.1-0.2 \%$ of the population [17]. Seven of $4244(0.16 \%)$ individuals in this study reported true oligodontia, also in keeping with the literature; thus, the self-reported nature of this study is a limitation but the reported values are consistent with other studies. In general, failure to form one or more permanent teeth (excluding third molars) is reported to be about twice as common in females as in males, and again, our data reflect that trend.

Overall, this study did not provide support for there being a general predisposition to missing teeth among a large cohort of CRC patients. It does not, however, rule out the likelihood that a small percentage of individuals may have germline variants in genes that predisposed them to both CRC and missing teeth. It may be that if a population of people with oligodontia were surveyed for cancer risks, one might still find that this is a marker for colorectal neoplasia but that oligodontia is so uncommon as to not be appreciable in a study of the size we conducted.

Of note, tooth agenesis is a common, multifactorial birth defect and can be sporadic or familial (inherited as dominant, recessive, or X-linked), and present variable penetrance in affected families [17]. It also presents variable prevalence rates among different ethnic backgrounds; therefore, the use of family-based studies may allow detection of segregation between different phenotypes and improve the likelihood of gene discovery in affected families.

AXIN2 mutations have been regarded as causative for the segregation of CRC and oligodontia in a Finnish family [3]. In the present study, we sequenced the AXIN2 gene locus in two probands presenting colon cancer and tooth agenesis (although the milder form, hypodontia) and having relatives with tooth agenesis. We found a common missense variant (P50S, rs2240308; minor allele frequency approximately 0.35 in North America) in one of the probands. Five of this proband's siblings including an unaffected sister (without tooth agenesis) also presented the variant. The AXIN2 P50S variant is unlikely related to the familial tooth agenesis or the colon cancer phenotype in this family. Additional, and yet possibly unknown genes, may be contributing to the segregation of these two different clinical phenotypes. Additional studies are underway to molecularly characterize the relevant genes in this population, with special attention to those few families that did report familial hypo- or oligodontia.

\section{Conclusion}

This study did not provide support for there being a general predisposition to missing teeth among a large cohort of CRC patients. The study does not exclude the possibility, however, that individuals presenting with notable hypodontia/oligodontia might still have an increased risk for colorectal neoplasia.

\section{Abbreviations}

APC: Adenomatous polyposis coli; Cl: Confidence interval; C-CFR: Colon cancer family registry; CRC: Colorectal cancer; OR: Odds ratio; WNT: Wingless/integration.

\section{Competing interests}

The authors declare that they have no competing interests.

\section{Authors' contributions}

NML conceived of the study. SG, NML, and DD collected the data. NML and AKW performed the data analysis. AL and RS conducted the sequencing of the AXIN2 gene. All authors read, critically reviewed, and approved the final manuscript.

\section{Acknowledgements}

We are grateful to the Colon Cancer Family Registry participants who provided this information along with so much more additional information over the years, and to Barbara J. Balgaard for her assistance in the preparation of this manuscript.

\section{Author details}

'Department of Health Sciences Research, Mayo Clinic, 13400 East Shea Blvd, Scottsdale, AZ 85259, USA. ${ }^{2}$ Centre for Molecular, Environmental, Genetic, and Analytic Epidemiology, University of Melbourne, Melbourne, VIC, Australia. ${ }^{3}$ Cancer Care Ontario, Toronto, ON, Canada. ${ }^{4}$ Department of Laboratory Medicine and Pathology, Mayo Clinic, Rochester, MN, USA. ${ }^{5}$ Department of Oral Biology and Center for Craniofacial and Dental Genetics, University of Pittsburgh, Pittsburgh, PA, USA. ${ }^{6}$ Departments of Endodontics, School of Dentistry, and Pediatric Research Center, Medical School, University of Texas Health Science Center, Houston, TX, USA.

Received: 21 January 2014 Accepted: 24 February 2014

Published: 10 March 2014

\section{References}

1. Nieminen P: Genetic basis of tooth agenesis. J Exp Zool B Mol Dev Evol 2009, 312B:320-342

2. Mitsiadis T, Luder $\mathrm{H}$ : Genetic basis for tooth malformations: from mice to men and back again. Clin Genet 2011, 80:319-329.

3. Lammi L, Arte S, Somer M, Jarvinen H, Lahermo P, Thesleff I, Pirinen S, Nieminen P: Mutations in AXIN2 cause familial tooth agenesis and predispose to colorectal cancer. Am J Hum Genet 2004, 74:1043-1050.

4. Renkonen ET, Nieminen P, Abdel-Rahman WM, Moisio AL, Jarvela I, Arte S, Jarvinen HJ, Peltomaki P: Adenomatous polyposis families that screen APC mutation-negative by conventional methods are genetically heterogeneous. J Clin Oncol 2005, 23:5651-5659.

5. Groen EJ, Roos A, Muntinghe FL, Enting RH, de Vries J, Kleibeuker JH, Witjes MJ, Links TP, van Beek AP: Extra-intestinal manifestations of familial adenomatous polyposis. Ann Surg Oncol 2008, 15:2439-2450.

6. Newcomb PA, Baron J, Cotterchio M, Gallinger S, Grove J, Haile R, Hall D, Hopper JL, Jass J, Le Marchand L, Limburg P, Lindor N, Potter JD, Templeton AS, Thibodeau S, Seminara D: Colon Cancer Family Registry: an international resource for studies of the genetic epidemiology of colon cancer. Cancer Epidemiol Biomarkers Prev 2007, 16:2331-2343.

7. Win AK, Young JP, Lindor NM, Tucker KM, Ahnen DJ, Young GP, Buchanan DD, Clendenning M, Giles GG, Winship I, Macrae FA, Goldblatt J, Southey MC, Arnold J, Thibodeau SN, Gunawardena SR, Bapat B, Baron JA, Casey G, Gallinger S, Le Marchand L, Newcomb PA, Haile RW, Hopper JL, Jenkins MA: Colorectal and other cancer risks for carriers and noncarriers from families with a DNA mismatch repair gene mutation: a prospective cohort study. J Clin Oncol 2012, 30:958-964.

8. Cleary SP, Cotterchio M, Jenkins MA, Kim H, Bristow R, Green R, Haile R, Hopper JL, LeMarchand L, Lindor N, Parfrey P, Potter J, Younghusband B, Gallinger S: Germline MutY human homologue mutations and colorectal cancer: a multisite case-control study. Gastroenterology 2009, 136:1251-1260.

9. Peterlongo P, Howe LR, Radice P, Sala P, Hong YJ, Hong SI, Mitra N, Offit K Ellis NA: Germline mutations of AXIN2 are not associated with nonsyndromic colorectal cancer. Hum Mutat 2005, 25:498-500.

10. Mostowska A, Biedziak B, Jagodzinski PP: Axis inhibition protein 2 (AXIN2) polymorphisms may be a risk factor for selective tooth agenesis. J Hum Genet 2006, 51:262-266.

11. Lejeune S, Guillemot F, Triboulet JP, Cattan S, Mouton C, Porchet N, Manouvrier S, Buisine MP: Low frequency of AXIN2 mutations and high 
frequency of MUTYH mutations in patients with multiple polyposis. Hum Mutat 2006, 27:1064.

12. Callahan N, Modesto A, Meira R, Seymen F, Patir A, Vieira AR: Axis inhibition protein 2 (AXIN2) polymorphisms and tooth agenesis. Arch Oral Biol 2009, $54: 45-49$.

13. Letra A, Bjork B, Cooper ME, Szabo-Rogers H, Deleyiannis FW, Field LL, Czeizel AE, Ma L, Garlet GP, Poletta FA, Mereb JC, Lopez-Camelo JS, Castilla EE, Orioli IM, Wendell S, Blanton SH, Liu K, Hecht JT, Marazita ML, Vieira AR, Silva RM: Association of AXIN2 with non-syndromic oral clefts in multiple populations. J Dent Res 2012, 91:473-478.

14. Letra A, Menezes R, Granjeiro JM, Vieira AR: AXIN2 and CDH1 polymorphisms, tooth agenesis, and oral clefts. Birth Defects Res A Clin Mol Teratol 2009, 85:169-173.

15. Menezes R, Marazita ML, Goldstein McHenry T, Cooper ME, Bardi K, Brandon C, Letra A, Martin RA, Vieira AR: AXIS inhibition protein 2, orofacial clefts and a family history of cancer. J Am Dent Assoc 2009, 140:80-84.

16. Marvin ML, Mazzoni SM, Herron CM, Edwards S, Gruber SB, Petty EM: AXIN2-associated autosomal dominant ectodermal dysplasia and neoplastic syndrome. Am J Med Genet A 2011, 155A:898-902.

17. Polder BJ, Van't Hof MA, Van der Linden FP, Kuijpers-Jagtman AM: A meta-analysis of the prevalence of dental agenesis of permanent teeth. Community Dent Oral Epidemiol 2004, 32:217-226.

doi:10.1186/1897-4287-12-7

Cite this article as: Lindor et al:: Colorectal cancer and self-reported tooth agenesis. Hereditary Cancer in Clinical Practice 2014 12:7.

\section{Submit your next manuscript to BioMed Central and take full advantage of:}

- Convenient online submission

- Thorough peer review

- No space constraints or color figure charges

- Immediate publication on acceptance

- Inclusion in PubMed, CAS, Scopus and Google Scholar

- Research which is freely available for redistribution 